\title{
Mini-symposium
}

\section{Modern management of myocardial infarction: introduction}

\begin{abstract}
t is not so very long ago that the treatment for a patient with myocardial infarction consisted of bed rest and analgesia. Myocardial destruction was observed but not modified by the cardiologist. The situation is very different at the start of the 2lst century. Decades of large randomised clinical trials have informed physicians of the benefits of both pharmacological and mechanical methods of reperfusion to the great benefit of patients with acute coronary occlusion. It has become increasingly clear that to make a real impact on the prognosis of patients with myocardial infarction, a wide perspective is needed and the greatest successes have occurred when whole health care delivery systems are integrated to provide care for patients throughout their entire illness, rather than concentrating on the narrow focus of coronary care unit and catheter laboratory treatment. The arguments over whether to use mechanical or pharmacological reperfusion are now being subsumed into the development of strategies where both are integrated to provide the greatest benefits to patients. It is evident that one strategy will not be universal and that there may be many different solutions that are applicable and optimal under different geographical and organisational situations.
\end{abstract}

There is no disagreement as to the vital importance of speed of reperfusion. In the first article in this mini-symposium, in an article discussing the prehospital care of patients with myocardial infarction, Welsh and Armstrong note that the time to treatment in major clinical trials has stalled at around three hours after symptom onset. The challenge for cardiologists is therefore much wider than hospital care, and treatment with thrombolytic agents can be started before the patient reaches hospital. This may be particularly effective in geographically remote situations. Treatment with pharmacological agents can also be used to "facilitate" treatment with primary angioplasty and this approach is discussed by Brodie in the second article. With increasing evidence that primary angioplasty is effective even in situations where patients require transfer from local hospitals to interventional cardiac centres, optimising the chances of successful reperfusion with "upstream" pharmacological agents may be the way forward.

The wholly justifiable focus on obtaining an open artery as soon as possible may have blinded cardiologists to the unfortunate fact that a significant minority of patients do not regain full myocardial perfusion despite the restoration of TIMI III flow. This subset of patients does badly as a result. In the third article, Prasad and Gersh outline the pathophysiology and diagnosis of myocardial malperfusion and discuss possible approaches to prevention and treatment.

Primary angioplasty for myocardial infarction remains an impressive and useful therapy, but the future will surely see improvements in the technique. Possible advances are discussed by Smith, Mathur, and Rothman in the last article. We may see attempts in the future to widen the scope of percutaneous intervention to encompass plaque stabilisation and myocardial repair as well as reperfusion.

Acute coronary occlusion should no longer be a major life event, provoking retirement from work and lifelong disability. We already have the means to deliver treatment that provides such rapid reperfusion and symptom relief that some patients are left wondering if they really had a heart attack. The goal over the next decade has to make this experience a reality for all patients with myocardial infarction.

Correspondence to: Dr Charles J Knight, Barts and the London Heart Centre, London Chest Hospital, Bonner Road, London E2 9JX, UK:

Charles.Knight@bartsandthelondon.nhs.uk

\section{IMAGES IN CARDIOLOGY}

\section{Crossing epicardial coronary arteries}

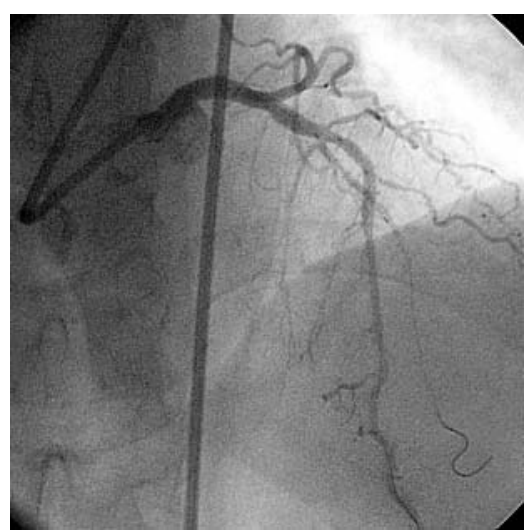

A 54 year old woman with diabetes and hypertension was admitted to the outpatient clinic complaining of chest pain of one year's duration. An ECG showed dynamic ST segment deviation compared with a previous ECG. Coronary angiography revealed an abnormality of the left anterior descending coronary artery (LAD). The LAD up to the diagonal and septal branch was normal, but then continued as two arteries (medial and lateral) crossing over each other. The medially arising artery was in diagonal artery territory, while the diagonal artery was in LAD territory. Both had a stenosis and were treated with intracoronary stent implantation. This anomaly of crossing coronary arteries has never been seen in our laboratory before, and only two reports on the anomaly have been previously published. 